\title{
New research shall be initiated in regard with the effects of mood alternation during neurological shutdown
}

\author{
PiMann Getsemi* \\ (ORCiD: 0000-0003-0579-8966) \\ The White House, 1600 Pennsylvania Ave \\ NW, Washington, DC 20500 \\ International Federation of Red Cross and \\ Red Crescent Societies, Geneva 19 Switzerland
}

January 25, 2019

\begin{abstract}
In 1994 the Death With Dignity Act was first passed by Oregon voters successfully, and it became the first law in American history permitting physician-assisted suicide. Pain and negative mental feelings and physical unconsciousness about the future events in the Universe are getting considered as the only disadvantages of the death. Aside of the issues of human cloning and mind uploading for making progress towards eternity of life, complementary researches in-regard-with the effects of mood alternation during neurological shutdown shall be initiated. Alternation on the human feelings by stimulation of the brain's synapses would lead to a more peaceful mental shutdown. The pain's circuit (the circuit of negative emotions during the death) shall be extracted and gets stimulated during the death. The stimulation of the circuit usually begins right after the act of suicide and suppression of the oxygen follow, and then gets followed for about 15 minutes. Since the Brain Research Initiative began in 2013, NIH and the Brain Research Initiative are responsible for further researches on a variety of different brain circuits. The BRAIN Initiative, is a collaborative, public-private research initiative announced by the Obama administration with the goal of supporting the development and application of innovative technologies that can create a dynamic understanding of brain function.
\end{abstract}

*Email address: peiman.ghasemi@aol.com - The White House and the IFRC wont be responsible to answer any questions. To send your comments, only use the (personal) email address above, please. 


\section{Neurological shutdown towards experience of a pain- less death}

The brain like all the other body organs needs some fuel to function. When we suppress the oxygen follow through inhalation of inert gases the brain stops to function. Some other chemicals may have some potentials to stop the synapses to work and transmit information through a same way also. In the next phase (when we use a slow method of suicide such as inhalation of gases) the hallucinations begin as a phase what we call the near death experience (NDE). Terrible hallucinations may surround us during the NDE. Monitoring the people's hallucinations via mind control advancements would help us to understand more about what is happening inside the brain in this phase of death.

Application of analgesic and also recreational drugs in combination with radio-frequencies (ELF, EHF, etc.) and mind control shall be initiated on patients during general anesthesia, before and during inhalation of inert-gases (such as nitrogen or helium) for NDE.[2] Alternation on the human feelings by stimulation of the brain's synapses would lead to a more peaceful mental shutdown. In this phase through using radio-frequencies we change the patients hallucinations to positive paranoia and to maximum joy.

Subsequent and one by one destruction of some brain circuits may be and only may be useful also. However there is a negative point that destruction of some parts of the brain while the other parts are working may cause a bad feeling for the patient and the whole brain. Hence we may pass through implication of this action. More importantly, there shall be no panic and sense of struggling anymore as we use inert gases or some other chemicals for the mental shutdown. And also for NDE the pain's circuit (and the circuit of negative emotions during the death) shall be extracted and gets stimulated during the death. Application of general anesthesia in combination with chemical enthusiastic elements which would make the body organs calm, maintaining the maximum rate of positive paranoia and imaginations through using radio waves or drug substances would be required. ELF and remote-sensing technologies have a potential for monitoring and reprograming visual hallucinations.[1] Even though implication of a state of unconsciousness and deep-sleep for every cells and body organs through using some chemicals (which function in a way similar to the local enthusiastic drugs) could be applicable.

Sudden death usually follows by inhalation of inert gases. By the progress of technology, following advancements on directed energy systems and magnetic resonance, over an introductory research on potentials of application of mood altering waves on the brain of the people during death procedure by inert gases under general anesthesia which can lead to a painless consecutive death, we shall investigate for the possibility of prevention of the extreme pain that a volunteer patient may highly suffer of, before the death as a state for relieving the pain itself. The basal ganglia, S1 and BA3, S2, BA46, BA10, BA9, BA5, the pretectal area, the hippocampus (and the other parts of the limbic system), and the thalamus are the most important locations in the human's brain, and targeting 
them destroys the home of our personality and the control/attention center of the brain, over NDE it leads to termination of life of a patient who is suffering of intolerable pain, but the patient won't sense any bad feelings at an ideal state. About the entire parts of the body, except of the brain itself, for natural properties of neurotransmitters in the human's nervous system, there would be a very small latency observable from the instantaneous moment of trauma until the pain gets deliver to the diencephalon to become sensible/understandable for a human. For example when someone accidentally drops down from a height and the one breaks his own bones, following to a neurological shock, instantaneously, just when the accident/trauma is happened this latency in a fraction of time is observable. Inside the skull, the meninges have pain receptors, and it's responding to noxious stimuli. However, the nervous structure of the brain is different, there are no pain receptors in cerebral cortex, and also the white matter, inside our brain; but meanwhile some reactions may be observable between two interactive parts of the brain for interpretation of bad feelings/pain signals. Therefore as an optimum complementary step, destruction process of the interactive areas (if required), subsequently, would get done over a small fraction of time (this could be including making small slices on or between such areas). In conclusion, distinct parts of the cerebrum and the thalamus are active locations of self-personality and attention. In elder patients of extreme pain who suffer of untreatable illnesses, it may get considered voluntary as a final decision by the victim and the victim's relatives. Thus, this experiment has several potentials for application; more research must be conducted in regard with in-depth understanding of mood alternation methods during neurological shutdown.

\section{What is an exit bag?}

In 1976 California Governor Edmund G. Brown Jr. signs the California Natural Death Act into law and California becomes the first state in the nation to grant terminally ill persons the right to authorize withdrawal of life. In 1994 the Oregon Death With Dignity Act is passed, becoming the first law in American history permitting physician-assisted suicide. Derek Humphry in collaboration with John Hofsess (Canadian writer and right to die activist), and engineers and physicians originally introduced the suicide bag with inert gas method which is also called the exit bag. It is a euthanasia device consisting of a large plastic bag with a drawcord used to commit suicide through inert gas asphyxiation. It is usually used in conjunction with a flow of an inert gas like helium or nitrogen, which prevents the panic, sense of suffocation and struggling before unconsciousness, known as the hypercapnic alarm response caused by the presence of high carbon dioxide concentrations in the blood.[3] 


\section{References}

[1] Robert C. Beck. Mood modification with elf magnetic fields: A preliminary exploration. Archaeus, 1978.

[2] Olaf Blanke. The neurology of consciousness. London Academic Publishers, 2009.

[3] Fiona Nitschke, Philip; Stewart. The peaceful pill handbook. 2016. 